\title{
Aspects of a Reliable Autonomous Navigation and Guidance System for an Unmanned Surface Vehicle
}

\author{
Rui Song \\ Department of Mechanical Engineering \\ University College London \\ London, UK \\ r.song.11@ucl.ac.uk \\ Wenwen Liu \\ Department of Mechanical Engineering \\ University College London \\ London, UK \\ w.liu.11@ucl.ac.uk
}

\author{
Yuanchang Liu \\ Department of Mechanical Engineering \\ University College London \\ London, UK \\ yuanchang.liu.10@ucl.ac.uk \\ Richard Bucknall \\ Department of Mechanical Engineering \\ University College London \\ London, UK \\ r.bucknall@ucl.ac.uk
}

\begin{abstract}
This paper describes a novel navigation and guidance (NG) system designed to address the issue of receiving unreliable navigational data considering an unmanned surface vehicles (USVs). In the NG system, a confidence rate determination method has been designed to identify the uncertainty of the acquired data. According to the confidence rate, the risks from inaccurate data can be properly analysed facilitating the system generating a more reliable guidance route. The route is calculated using a newly developed algorithm named the constrained FM*. The new NG system has been verified in simulation environments with results proving the effectiveness and capabilities of the system.
\end{abstract}

Keywords—confidence level; fast marching*; path planning; USV

\section{INTRODUCTION}

Currently, there is an increasing interest in the design and development of cost-effective unmanned surface vehicles (USVs). USVs can be applied in both military and civilian tasks, such as weapons delivery, force multipliers, bathymetric survey and environmental monitoring. In order to make the USVs capable of autonomously undertaking these missions, a robust and reliable navigation and guidance (NG) system is required.

The NG system is responsible for accurate positioning and safe navigation of the USV and should be capable of dealing with sensor noises in regardless of environment. To achieve this, Kalman filtering (KF) has been widely used to improve the signal accuracy [1], [2] and [3]. In [4], the KF was applied to fuse multiple measurements from different sensors to give a more accurate position information of the USV. However, it remains unknown that how much the accuracy can be improved without evaluating the performance of the KF. Hence, in this paper, a novel confidence rate determination algorithm is developed to numerically describe the accuracy improvement when using the KF.

Based upon the processed navigational information, the NG system is able to generate a guidance route for the USV. To calculate an optimal trajectory, the implementation of deterministic path planning algorithms, such as A* algorithm [5] and fast marching (FM) method [6] is becoming more popular than the stochastic algorithms, such as the genetic [7] and ant colony [8] optimisation algorithms. The FM method has the benefit that can generate a path with improved consistency, completeness and continuity [9]. In order to promote the application on unmanned platforms, especially on USV platform, a number of improvements have also been made on the FM. [9] developed a FM square (FM ${ }^{2}$ ) path planning algorithm for robotic application. The algorithm can enlarge the obstacle areas to improve the safety of the trajectory. Furthermore [10] improves the $\mathrm{FM}^{2}$ algorithm by combining it with the $\mathrm{A}^{*}$ algorithm to save the computation time. The new algorithm is named as $\mathrm{FM}^{2}$ star $\left(\mathrm{FM}^{2 *}\right)$ algorithm. In [11] a constraint $\mathrm{FM}^{2}$ method is developed for USVs to solve the path planning with moving obstacles.

However, these algorithms do not give a detailed explanation of when and how to expand the obstacle areas. Obstacle area may be enlarged when there is no collision risk. In addition, the reliability of the navigational data is not considered for the expansion. To solve these problems, in this paper, a new risk assessment strategy is particularly developed and integrated into the $\mathrm{FM}^{2 *}$ algorithm to define the scale of expansion.

This paper is organised as follows. Section II describes the structure of the NG system. Section III explains the proposed methods determining the confidence rate. Section IV details the path searching algorithm based upon the FM* method. Results are shown in Section V with discussions. This paper is concluded in Section VI. 


\section{NAVIGATION AND GUIDANCE SYSTEM}

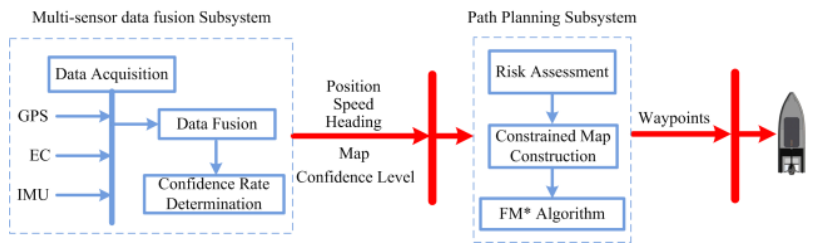

Fig. 1 USV navigation and guidance system.

The structure of the navigation and guidance (NG) system is shown in Fig. 1. It consists of two modular subsystems: 1) multisensor data fusion module (MDFM) and 2) path planning module (PPM). The two modules share different responsibilities to complete the USV navigation. The MDFM is designed to obtain the real time navigational data such as position, speed and heading of a USV. Data are processed through data fusion techniques with the associated data reliability evaluated. Fused data and the corresponding reliability value are then transmitted to the PPM together with the environment map. In the PPM, the risk of the unreliable data and obstacles is first assessed. According to the assessment, an environment map will be transformed into a grid map containing information of obstacle and free areas, which can be further converted to a constrained map with enlarged obstacle areas. The fast marching* $\left(\mathrm{FM}^{*}\right)$ method is subsequently applied upon the constrained map to generate a safe trajectory.

\section{MULTI-SENSOR DATA FUSION}

\section{A. Data acquisition}

As shown in Fig. 1, a GPS receiver, an Inertial Measurement Unit (IMU) and an electronic compass (EC) are installed on the NG system to provide USV's navigational data. The GPS and EC can provide absolute measurements of USV's position and heading while the IMU that is composed of an accelerometer and a gyroscope measures the acceleration and rotation of a USV's movement, respectively. Note that the readings from different sensors have different formats and should be converted into the same coordinate frame and time step [12].

\section{B. Data fusion}

Since the navigational sensors are associated with various noises making the obtained measurements inaccurate, the Kalman filtering (KF) algorithm can be used as an optimal estimator to improve the accuracy.

In general, the KF operation involves two updates: the time update and the measurement update as shown in Fig. 2. With the initial estimation of the state vector $x$ and its error covariance matrix $P$, the first predicted state $\hat{\boldsymbol{x}}^{-}$of system can be calculated by a system state model. The KF will then estimate the optimal state $\widehat{\boldsymbol{x}}$ by applying the sensor measurement $\mathbf{z}$. The estimation process is called the measurement update. After the estimation, the system will update its $P$ and enter into the next state to make a new prediction, and the prediction process is called the time update. This prediction-estimation process iterates the system and reduces the system error covariance to obtain an optimal state [13].

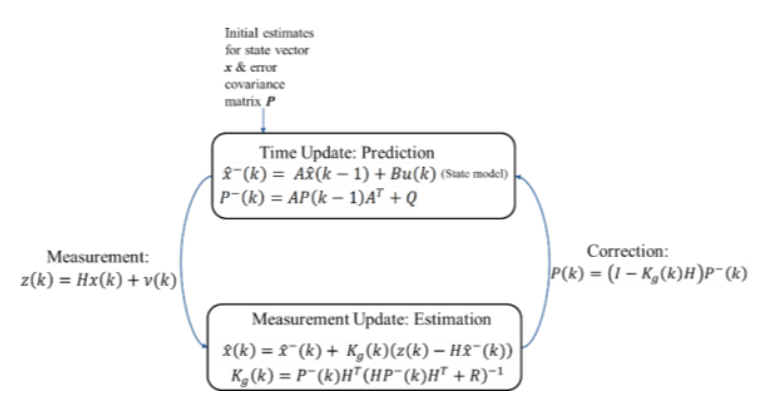

Fig. 2 Kalman filtering process

A KF based algorithm has been implemented in [4] to estimate USV's dynamic positions. In order to better represent the vehicle's motion, the algorithm adds USV's acceleration $(\boldsymbol{a})$ into the system state model to predict positions and obtains the absolute position measurements $(\boldsymbol{z})$ from GPS signal. The expressions giving the system state and measurement model are shown in (1) as:

$$
\left\{\begin{array}{c}
\boldsymbol{x}(k)=\left[\begin{array}{cccc}
1 & 0 & T_{s} & 0 \\
0 & 1 & T_{s} & 0 \\
0 & 0 & 1 & 0 \\
0 & 0 & 0 & 1
\end{array}\right] \boldsymbol{x}(k-1)+\left[\begin{array}{cc}
\frac{T_{s}^{2}}{2} & 0 \\
0 & \frac{T_{s}^{2}}{2} \\
T_{s} & 0 \\
0 & T_{s}
\end{array}\right] \boldsymbol{a}(k)+\boldsymbol{w}(k-1) \\
\boldsymbol{z}(k)=\left[\begin{array}{cccc}
1 & 0 & 0 & 0 \\
0 & 1 & 0 & 0
\end{array}\right] \boldsymbol{x}(k)+\boldsymbol{v}(k)
\end{array}\right.
$$

where $\boldsymbol{x}(k)$ denotes the system state vector at time step $k$ and has the form of $\left[\begin{array}{llll}p_{x} & p_{y} & v_{x} & v_{y}\end{array}\right]^{T} .\left(\begin{array}{ll}p_{x} & p_{y}\end{array}\right)$ and $\left(v_{x} \quad v_{y}\right)$ are the vehicle's position coordinates and velocities in $\mathrm{x}$ axis and $\mathrm{y}$ axis, respectively. $\boldsymbol{w}(k-1)$ and $\boldsymbol{v}(k)$ are the process and observation noises respectively, both of which can be expressed as the zero mean Gaussian white noise.

\section{Confidence rate determination}

During the process of KF, three components can be obtained in each iteration $k$ :

1) the predicted position: $\boldsymbol{\mu}_{\boldsymbol{p}}=\left(\hat{\boldsymbol{x}}^{-}(1,1) \quad \hat{\boldsymbol{x}}^{-}(2,1)\right)$,

2) the measured position: $\boldsymbol{\mu}_{\boldsymbol{m}}=(\mathbf{z}(1,1) \quad \boldsymbol{z}(2,1))$, and

3) the fused position: $\boldsymbol{\mu}_{\boldsymbol{f}}=(\widehat{\boldsymbol{x}}(1,1) \quad \widehat{\boldsymbol{x}}(2,1))$.

These three positions can be evaluated with regard to the true position of the USV by comparing the position error values.

Due to the nature of the KF, the position distributions of these three components can be described using the Gaussian probability density functions (pdf) given by (2), (3) and (4), respectively, where $\boldsymbol{r}$ denotes the true position of the USV.

$$
\begin{gathered}
\boldsymbol{y}_{\boldsymbol{p}} \triangleq \frac{1}{\sqrt{(2 \pi)^{2}\left|\Sigma_{p}\right|}} \exp \left(-\frac{1}{2}\left(\boldsymbol{\mu}_{\boldsymbol{p}}-\boldsymbol{r}\right)^{\mathrm{T}} \Sigma_{\boldsymbol{p}}^{-1}\left(\boldsymbol{\mu}_{\boldsymbol{p}}-\boldsymbol{r}\right)\right) \\
\boldsymbol{y}_{\boldsymbol{m}} \triangleq \frac{1}{\sqrt{(2 \pi)^{2}\left|\Sigma_{\boldsymbol{m}}\right|}} \exp \left(-\frac{1}{2}\left(\boldsymbol{\mu}_{\boldsymbol{m}}-\boldsymbol{r}\right)^{\mathrm{T}} \Sigma_{\boldsymbol{m}}^{-1}\left(\boldsymbol{\mu}_{\boldsymbol{m}}-\boldsymbol{r}\right)\right) \\
\boldsymbol{y}_{\boldsymbol{f}} \triangleq \frac{1}{\sqrt{(2 \pi)^{2}\left|\Sigma_{f}\right|}} \exp \left(-\frac{1}{2}\left(\boldsymbol{\mu}_{\boldsymbol{f}}-\boldsymbol{r}\right)^{\mathrm{T}} \Sigma_{\boldsymbol{f}}^{-1}\left(\boldsymbol{\mu}_{\boldsymbol{f}} \boldsymbol{r}\right)\right)
\end{gathered}
$$


$\Sigma_{p}$ is the position error covariance matrix before fusion, $\Sigma_{m}$ is the covariance matrix representing the uncertainty associated with the measurements and $\Sigma_{f}$ is the position error covariance matrix after fusion. $\Sigma_{p}, \Sigma_{p}$ and $\Sigma_{f}$ are expressed in the form of (5), where $\sigma_{p}, \sigma_{m}$ and $\sigma_{f}$ are the corresponding variances with $\boldsymbol{r}$. The corresponding position distributions of the three components are plotted as shown in Fig. 3.

$$
\begin{aligned}
\Sigma_{p} & =\left[\begin{array}{cc}
\sigma_{p}{ }^{2}(1,1) & 0 \\
0 & \sigma_{p}{ }^{2}(1,2)
\end{array}\right] \\
\Sigma_{\boldsymbol{m}} & =\left[\begin{array}{cc}
\boldsymbol{\sigma}_{\boldsymbol{m}}{ }^{2}(1,1) & 0 \\
0 & \boldsymbol{\sigma}_{\boldsymbol{m}}{ }^{2}(1,2)
\end{array}\right] \\
\Sigma_{f} & =\left[\begin{array}{cc}
\sigma_{f}^{2}(1,1) & 0 \\
0 & \sigma_{f}{ }^{2}(1,2)
\end{array}\right]
\end{aligned}
$$
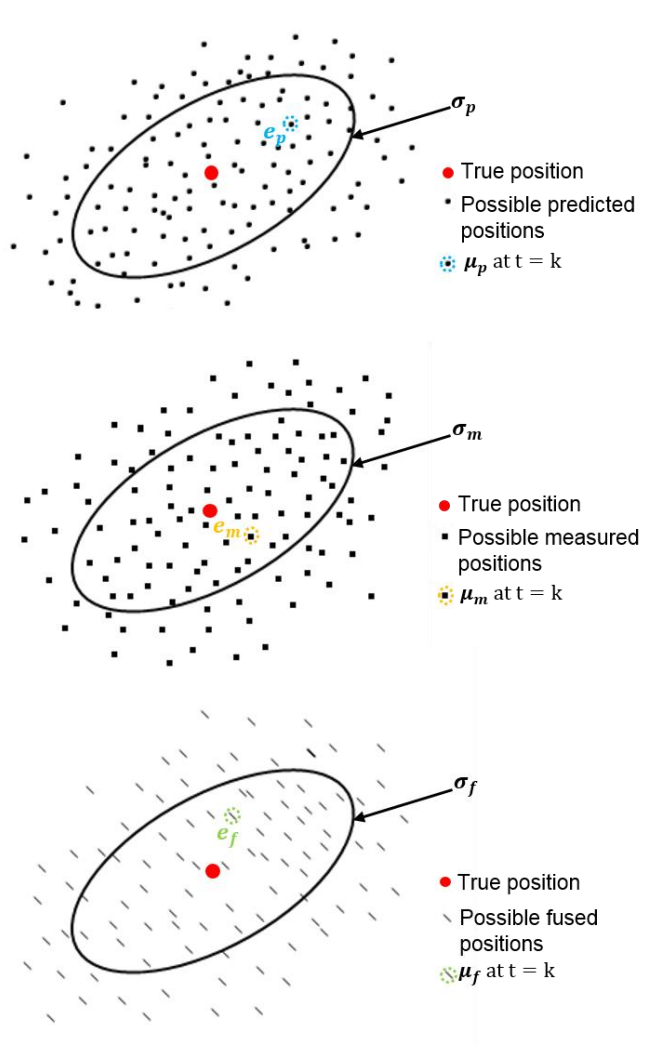

Fig. 3 Position distributions

At each time $k$, the obtained $\mu_{p}, \mu_{m}$ and $\mu_{f}$ will have fixed deviations from the true position denoted as $\boldsymbol{e}_{\boldsymbol{p}}, \boldsymbol{e}_{\boldsymbol{m}}$ and $\boldsymbol{e}_{f}$, respectively. The relationships between $\mu, \boldsymbol{e}$ and $\boldsymbol{r}$ can be expressed as:

$$
\begin{aligned}
\mu_{p} & =r+e_{p} \\
\mu_{m} & =r+e_{m} . \\
\mu_{f} & =r+e_{f}
\end{aligned}
$$

Since the value of the deviations and the true position are unknown in practical applications, the differences between the three components $\mu_{p}, \mu_{m}$ and $\mu_{f}$ are therefore used to verify the working performance of the KF, named as position precision $\Delta \boldsymbol{S}$. The values of $\Delta \boldsymbol{S}$, which are equivalent to the differences

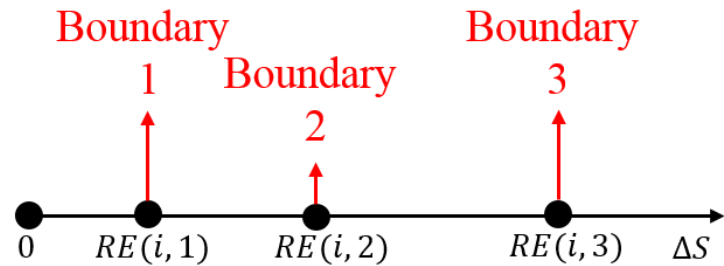

Fig. 4 Position precision with defined ranges

between the deviations, can be calculated as expressed in (7). The smaller the value of $\Delta \boldsymbol{S}$, the better the performance. For example, if $\Delta S(1)$ is small, it means the predicted position and the measured position are very close, and the values of $\mu_{p}$ and $\mu_{m}$ can be more trusted. The values of $\Delta S$ are used to determine the confidence rate. Each $\Delta S$ are divided into four ranges as shown in Fig. 4 with three boundaries. The boundaries are denoted as $\boldsymbol{R} \boldsymbol{E}(i, \mathrm{j})$ and can be expressed as in (8).

$$
\begin{gathered}
\Delta S=\left[\begin{array}{c}
\Delta S(1) \\
\Delta S(2) \\
\Delta S(3)
\end{array}\right]=\left[\begin{array}{c}
\left|\mu_{m}-\mu_{p}\right| \\
\left|\mu_{f}-\mu_{p}\right| \\
\left|\mu_{f}-\mu_{m}\right|
\end{array}\right]=\left[\begin{array}{c}
\left|e_{m}-e_{p}\right| \\
\left|e_{f}-e_{p}\right| \\
\left|e_{f}-e_{m}\right|
\end{array}\right], \\
\boldsymbol{R E}(i, j)=\left[\begin{array}{ccc}
\left|\sigma_{p}-\sigma_{m}\right| & \left|\sigma_{p}+\sigma_{m}\right| & 2\left|\sigma_{p}+\sigma_{m}\right| \\
\left|\sigma_{f}-\sigma_{p}\right| & \left|\sigma_{f}+\sigma_{p}\right| & 2\left|\sigma_{f}+\sigma_{p}\right| \\
\left|\sigma_{f}-\sigma_{m}\right| & \left|\sigma_{f}+\sigma_{m}\right| & 2\left|\sigma_{f}+\sigma_{m}\right|
\end{array}\right] \\
i, j=1,2,3
\end{gathered}
$$

Based on the range of the $\Delta S$, the rules shown in Table I are established to determine the grade of the confidence rate $(C R)$. As shown in Fig. 5, for each $\Delta S(i)$, the $C R(i)$ value can be computed according to the grade. An overall $C R$ of the navigational data can then be determined by assigning a weighting to each $C R(i)$ as illustrated in Fig. 5.

TABLE I. CONFIDENCE RATE DETERMINATION RULES

\begin{tabular}{ll}
\hline Rule & $\boldsymbol{C R}$ Grade \\
\hline 1 & If $(\Delta \boldsymbol{S}(i) \leq \boldsymbol{R} \boldsymbol{E}(i, 1)$, then $C R(i)$ is good \\
2 & If $(\boldsymbol{R} \boldsymbol{E}(i, 1)<\Delta \boldsymbol{S}(i) \leq \boldsymbol{R} \boldsymbol{E}(i, 2)$, then $C R(i)$ is normal \\
3 & If $(\boldsymbol{R} \boldsymbol{E}(i, 2)<\Delta \boldsymbol{S}(i) \leq \boldsymbol{R} \boldsymbol{E}(i, 3)$, then $C R(i)$ is bad \\
4 & If $(\Delta \boldsymbol{S}(i)>\boldsymbol{R} \boldsymbol{E}(i, 3)$, then $C R(i)$ is worst \\
\hline
\end{tabular}

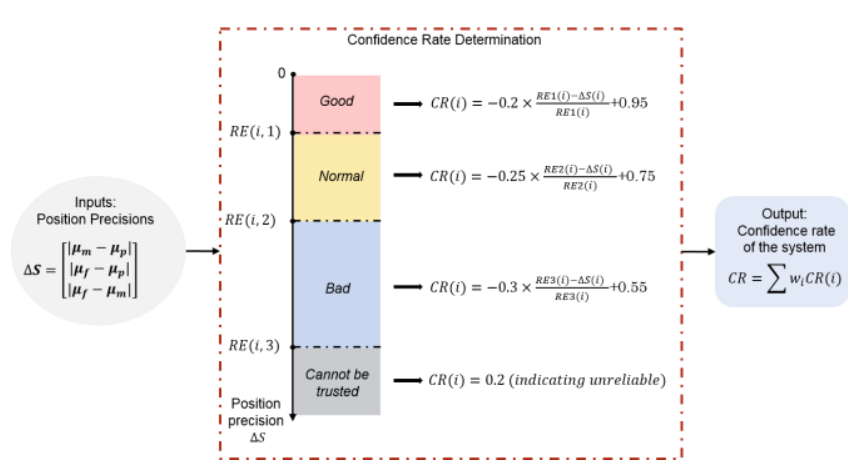

Fig. 5 Schematic of $C R$ value determination 


\section{CONSTRAINED FM* PATH PLANNING}

\section{A. Problem statement}

When planning the path, the environment map is converted into a uniform configuration space (C-space). In the $\mathrm{C}$-space, the obstacle area is denoted as $C_{o b s}$ and on the contrary, $C_{\text {free }}$ denotes the free space. The USV is represented as a point in the C-space, and its movement properties are expressed as a configuration, $q$ $=\langle x, y, \phi, v\rangle$. The $q$ specifies the state of USV at location $p(x, y)$ with $\phi$ representing the USV's heading angle and $v$ for the speed. When navigating in a time varying environment, the state of USV can be represented as $s=\langle q, t\rangle$ with an additional time domain added. Given a $C$-space $\Omega$, the path planning problem is to find a curve (or trajectory)

$$
\sigma:[0,1] \rightarrow C_{\text {free }}, \quad c \mid \rightarrow \sigma(c),
$$

where $c$ is the arc length of $\sigma$. An optimal trajectory should minimise a set of both internal and external constraints, such as computation time, fuel consumption (distance) and danger. The constraints can be described in a cost function $\tau$ represented in (10), which depends on the state of USV, s. Specifically, in this paper the uncertainty of received data information is taken into account when updating $\tau(s)$.

$$
\tau . \Omega \rightarrow \mathrm{R}+, \quad s \mid \rightarrow \tau(s), \tau(s)>0
$$

\section{B. $F M^{*}$ method}

To generate a safe path with high computational efficiency, the conventional $\mathrm{FM}^{*}$ method is selected as the base path searching method. The conventional $\mathrm{FM}^{*}$ method is first introduced by [14]. It is a hybrid path searching method that combines the $\mathrm{A}^{*}$ algorithm [15] and the fast marching (FM) method [16]. Similar to the $A^{*}$ algorithm, it uses heuristic to drive the search of possible path. The heuristic is a weighted distance that remains to be marched to the goal point. A parameter $\lambda$, ranging from 0 to $100 \%$, is introduced in the cost function as represented in (11) to represent the weight of heuristic influence.

$$
\tau(s)=U(s)+\lambda V(s),
$$

where $U(s)$ represents the traversed distance cost from the start point to the current point of USV. While $V(s)$ is the estimated distance cost from the current point to the goal point.

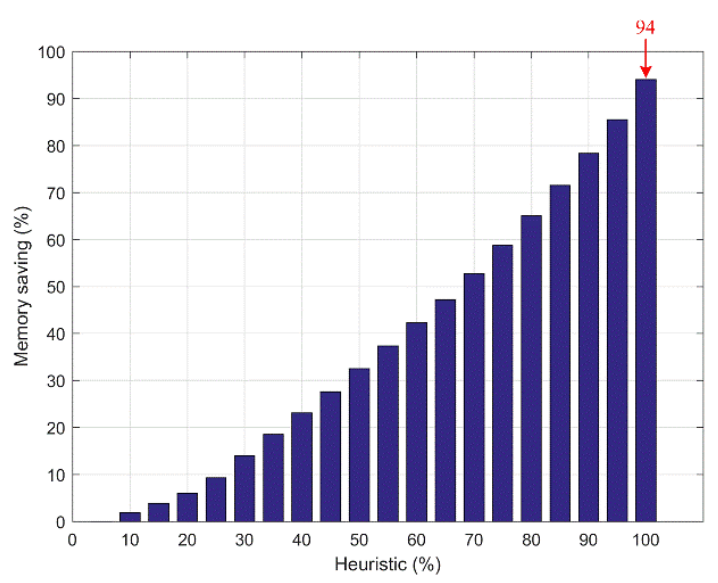

Fig. 6 Memory saving comparisons with different $\lambda$ values. The amount of memory saving increases as the value of increases. $94 \%$ of computation memory space can be saved when $\lambda$ equals to $100 \%$.

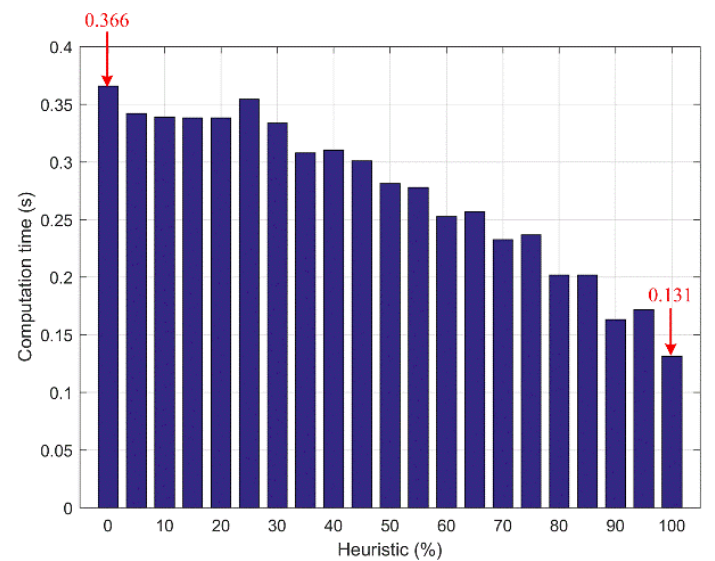

Fig. 7 Computation time comparisons with different $\lambda$ values in an environment with $500 * 500$ grids. The longest time is $0.366 \mathrm{~s}$ when $\lambda=0$, which consumes almost 3 times computational time than the time when $\lambda=100 \%(0.131 \mathrm{~s})$.

The effects of $\lambda$ have been verified against computing memory saving and computational time as displayed in Fig. 6 and Fig. 7 respectively. When $\lambda$ equals to 0 , which means there is no heuristic guiding, the $\mathrm{FM}^{*}$ runs as the same as the FM method. When $\lambda$ increases, the computer memory space decreases as well as the computational time. Based on these evaluations, the value of $\lambda$ is set as $100 \%$ when developing the constrained $\mathrm{FM}^{*}$ path planning algorithm.

\section{Constrained $F M^{*}$ path planning}

The flow chart of the constrained FM* algorithm is shown in Fig. 8. It takes three procedures for the constrained $\mathrm{FM}^{*}$ algorithm to generate a trajectory, which are 1) risk assessment, 2) constrained map construction and 3 ) waypoints generation. 


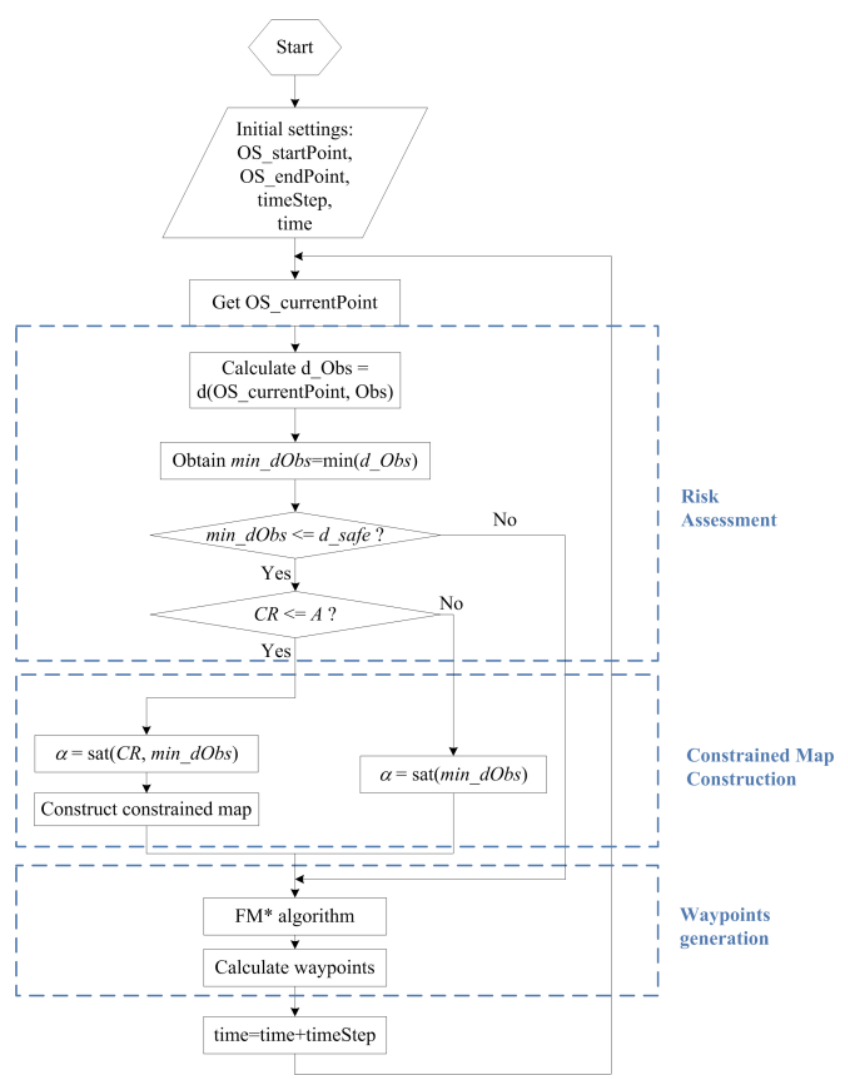

Fig. 8 Flow chart of the constrained FM* path planning algorithm.

\section{1) Risk assessment}

A new risk assessment strategy is designed to evaluate the overall risk that might affect the USV navigation, as depicted in Fig. 9. In this strategy, not only the risk from obstacles, but the risk generated from unreliable data are considered. The weightings of these two risks are denoted as $w(\mathrm{Obs})$ and $w(C R)$ respectively, and can be calculated as:

$$
\begin{gathered}
w(O b s)=\left(d \_s a f e-m i n \_d O b s\right) / d \_s a f e, \\
\text { and } w(C R)=(A-C R) / A,
\end{gathered}
$$

where $C R$ is the value of confidence rate obtained from the MDFM. $A$ is a predefined tolerant value of CR. d_safe is a predefined safe distance to obstacles, and min_dObs is the minimum distance between USV and obstacles in the environment of interest.

According to the values of $w(O b s)$ and $w(C R)$, a constraint safety factor $(\alpha)$ is introduced to indicate the overall risk of the surrounding environment. As shown in Fig. 9, the overall risk can be divided into three grades:

\section{Grade I:}

$w(O b s)<T o \_O b s \& \& w(C R)<T o \_C R$

\section{Grade II:}

$w(O b s)<T o \_O b s \& \& w(C R)>T o \_C R, O R$

$w(O b s)>T o \_O b s \& \& w(C R)<T o \_C R$

Grade III:
$w(O b s)>T o \_O b s \& \& w(C R)>T o \_C R$,

where To_Obs and To_CR are two predefined thresholds for $w(\mathrm{Obs})$ and $w(C R)$. Based on the grade of risks, the value of $\alpha$ can be defined as shown in Table II, where the Grade is denoted as RA, $\alpha_{\text {small }}$ and $\alpha_{\text {large }}$ are two predefined safety constraints. Note that RA equalling 0 represents no risk, the value of $\alpha$ is therefore 0 .

2) Constrained map construction and waypoints generation

The environment map $(M)$ is first transformed into a grid map using the Otsu image processing method [17], where the grid value of $\forall q \in C_{o b s}$ is 0 and $\forall q \in C_{\text {free }}$ is 1 . With the aim of saving the distance cost, the grid map is updated and reconstructed into a new constrained map $(M o)$ only when there is a potential collision risk, namely $\min \_d O b s<d \_s a f e$. Mo can be represented as shown in (14). The area of $C_{o b s}$ is enlarged according to $\alpha$ using the method in [9] and the new obstacle space is denoted as $C_{\text {obs_new. }}$

$$
M o=C_{\text {obs_new }} \cap C_{\text {free }}
$$

The FM* method is applied upon the Mo to generate a potential map (Wo) where each potential value represents the distance to the USV's target point.The gradient descent method is then applied on the Wo to calculate the trajectory.

TABLE II. DEFINITION OF CONSTRAINED SAFETY FACTOR

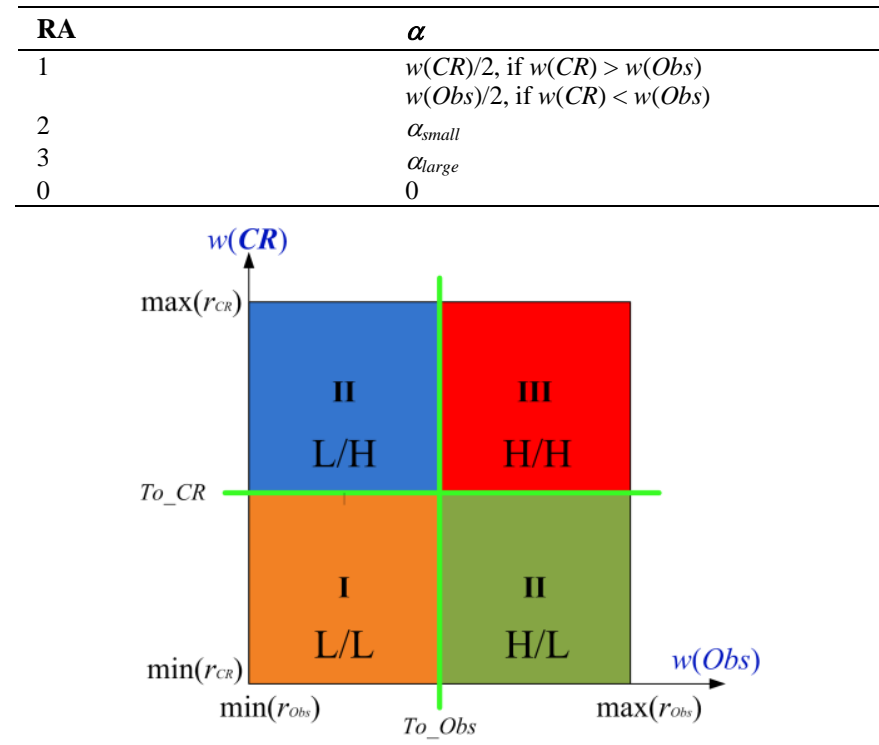

Fig. 9 Overall risk assessment strategy 


\section{RESUlTS}

Fig. 10 shows the simulation results to validate the proposed NG system. The USV is guided by the system from the start point to the end point in a time varying marine environment. Fig. 10 (b) shows the grid map representation of a water area near Plymouth harbour, with the size of $500 * 500$ pixels and each pixel representing $4 \mathrm{~m}$. Fig. 10(a), (c), (e), (g), (i) and (k) show the path planning results at time step 1, 6, 11, 16, 21 and 25. The blue line is the off-line trajectory before the mission started. Red lines are the online re-planned trajectories at different time steps. The light blue line is the USV tracking trajectory with USV's current location depicted in black circle. USV's start and goal points are represented in magenta dot and star respectively. Fig. 10(b),(d),(f),(h),(j) and (l) represent the corresponding constrained map. The colour from black to white represents the distance to obstacles. The darker the colour is, the closer the distance to the obstacle. The parameters settings of the simulation are listed in Table III; whereas, the values of $C R$, min_dObs and $\alpha$ are shown in Table IV.

It can be observed from Fig. 10(a)-(d) that before the USV approaching to the middle island, the updated trajectories do not deviate from the blue trajectory (off-line trajectory) as there is no collision risk ( $m i n \_d O b s>40$ pixel). However, after time step 11, the min_dObs becomes less than 40 pixels, and the algorithm starts to calculate the constraint safety factor. As shown in Table IV, the overall risks at time steps 11, 16 and 21 are ranked in RA 1 , and $\alpha$ are consequently calculated as 0.077 , 0.140 and 0.017. It also can be observed in Fig. 10(e)-(j) that the obstacle areas have been expanded accordingly and making generated trajectories keep a safe distance away from obstacles.

By comparing the results at in Fig. 10(g) and (e), $C R$ value at time step 16 is smaller meaning the acquired information is less reliable. $\alpha$ is therefore increased to make the updated trajectory farther away from the middle island. It also should be noted that in Fig. 10(k) and (1), when the USV gets close to the end point, a new $d \_s a f e$ is defined as 10 pixels. The value of $\alpha$ is calculated accordingly as 0.120 , which is larger than the value at time step 21 . Such a process is used to assure the safety of USV when approaching the end point area, where less free spaces are available.

These results indicate that the newly developed NG system can successfully evaluate the potential risk and ensure the safety of the generated trajectory in the situation where the data is unreliable.

TABLE III. InITIAL SETTINGS OF SimULATION

\begin{tabular}{ll}
\hline Parameters & Values \\
\hline Start point $\left(p_{0}\right)$ & $(141,113)$ \\
End point $\left(p_{g}\right)$ & $(212,453)$ \\
Speed & $1.5 \mathrm{~m} / \mathrm{s}$ \\
Heading & $60^{\circ}$ \\
d_safe $\left(\right.$ far from $\left.p_{0}\right)$ & 40 pixels \\
d_safe $\left(\right.$ far from $\left.p_{g}\right)$ & 10 pixels \\
A & $75.00 \%$ \\
$\max \left(r_{C R}\right)$ & $20.00 \%$ \\
$\min \left(r_{C R}\right)$ & $95.00 \%$ \\
To_Obs & $50.00 \%$ \\
To_CR $C R$ & $50.00 \%$ \\
$\alpha_{\text {small }}$ & 0.500 \\
$\alpha_{\text {large }}$ & 0.800 \\
Time interval & 120 \\
\hline
\end{tabular}

TABLE IV. VALUES OF CONSTRAINTS

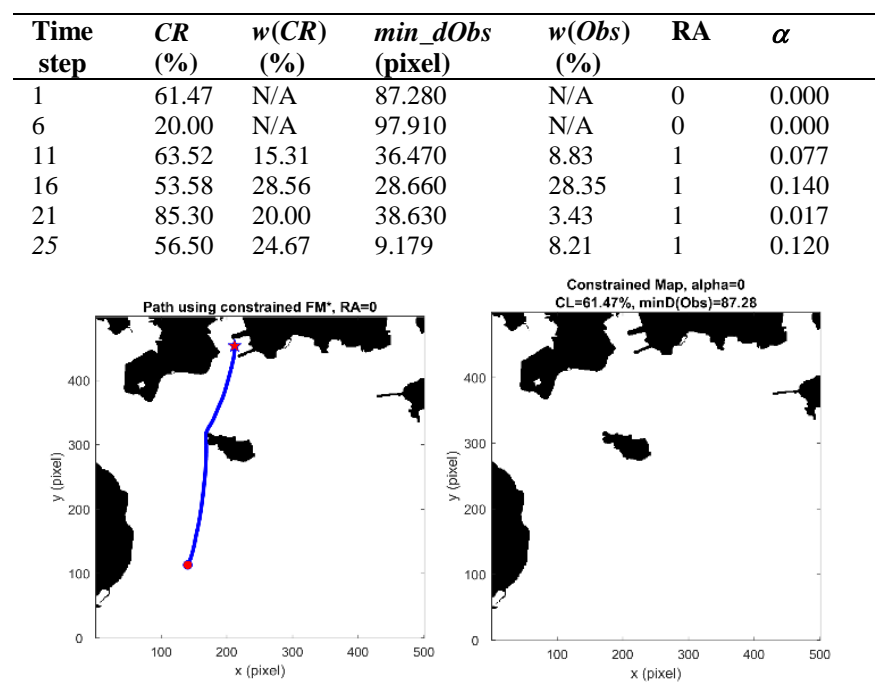

(a)

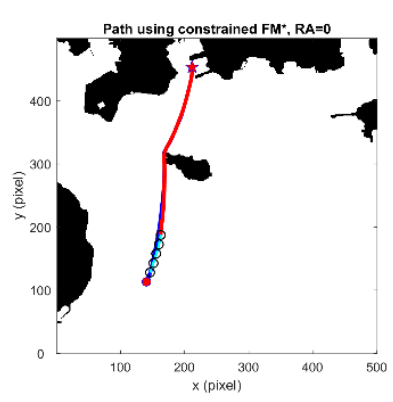

(c) (b)

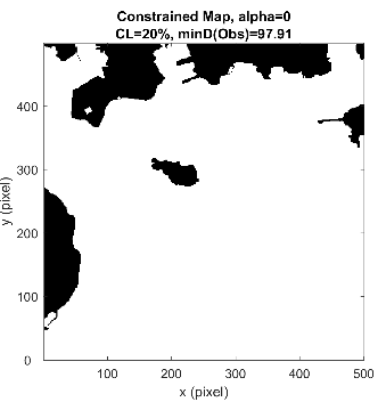

(d) 


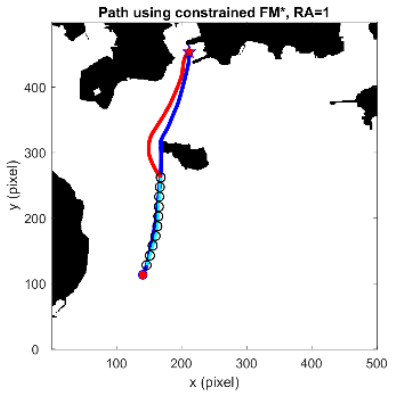

(e)

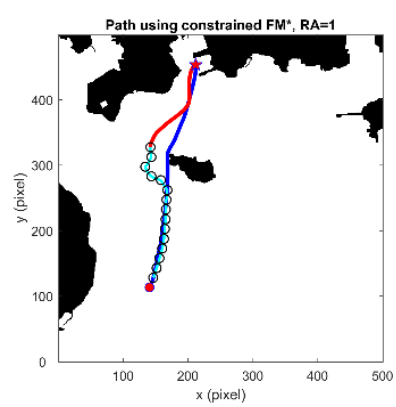

(g)

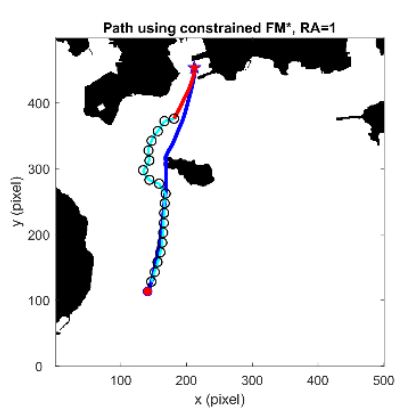

(i)

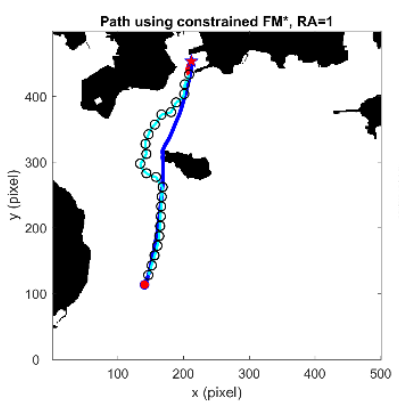

(k)

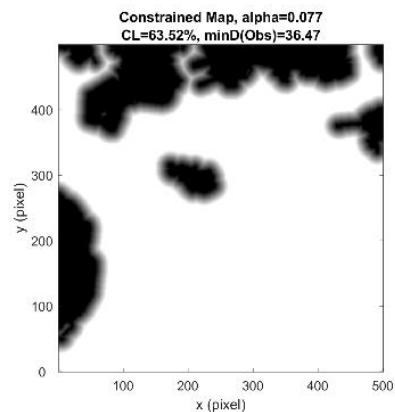

(f)

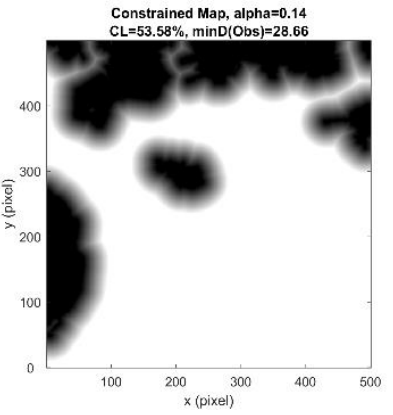

(h)

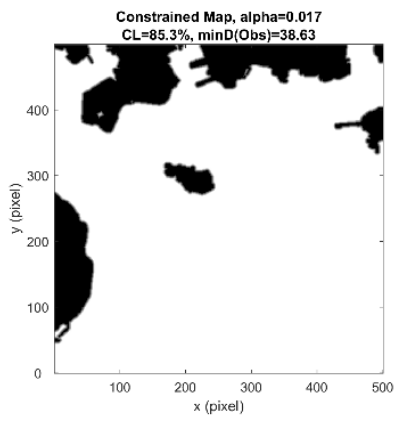

(j)

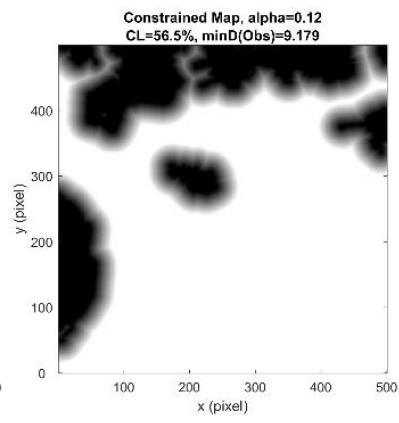

(1)
Fig. 10 Constrained FM* path planning results. (a),(c),(e),(g),(i) and (k) show the generated trajectories, where the blue line is the trajectory when USV locates in the start position; red lines are the updated trajectories at time 1,6,11,16,21 and 25 with USV's locations are depicted in black circles; light blue line is the USV tracking trajectory; USV's start and goal points are represented in magenta dot and star respectively. (b),(d),(f),(h),(j) and (l) represent the constrained map. The value of $C R$ and min_dObs are shown in the top of each subfigures

\section{CONCLUSION}

A NG system has been developed for practical applications, when the navigational data is unreliable. In the proposed system, the multi-sensor data fusion module calculates the position precision values to define the overall confidence rate of the navigational data. The path planning module of the system evaluates the risks according to the confidence rate and the minimum distance to obstacles, and generates an overall constrained safety factor for obstacle area expansion.

The simulation results show that the two subsystems can cooperate well and generate an optimal trajectory in a time varying environment. By optimising the three constraints of safety, distance and computation costs, the NG system guides the USV to follow the off-line planned trajectory and take evasive manoeuvers as soon as the potential collision risk is detected. The NG system can guide the USV to keep a farther distance away from obstacles when the confidence rate is below its tolerant value. Further improvements will be made to implement the NG system for moving obstacles avoidance.

\section{ACKNOWLEDGMENT}

This work is supported by the ACCeSS group. The Atlantic Centre for the innovative design and Control of Small Ships (ACCeSS) is an ONR-NNRNE programme with Grant no. N0014-03-0160, the group consists of universities and industry partners conducting small ships related researches.

\section{REFERENCES}

[1] Caccia, M., Bibuli, M., Bono, R., \& Bruzzone, G. (2008). Basic navigation, guidance and control of an unmanned surface vehicle. Autonomous Robots, 25(4), 349-365.

[2] Naeem, W., Xu, T., \& Sutton, R. (2009, June). An intelligent integrated navigation and control solution for an unmanned surface craft. In Signals and Systems Conference (ISSC 2009), IET Irish (pp. 1-6). IET.

[3] Motwani, A. (2015). Interval Kalman Filtering Techniques for Unmanned Surface Vehicle Navigation

[4] Liu W., Liu Y., Song R. and Bucknall R. (2015). 'Towards the development of an autonomous navigation system for unmanned vessels'. Proceedings of International Navigation Conference. February 24th-26th 2015, Manchester Conference Centre/UK.

[5] Song, R. (2014). Path Planning and Bi-directional Communication for Unmanned Surface Vehicle. MIDAS Technical Report, MIDAS MSE. 2014. TR. 011.

[6] Xu, B., Stilwell, D. J., \& Kurdila, A. J. (2013). Fast path re-planning based on fast marching and level sets. Journal of Intelligent \& Robotic Systems, 71(3-4), 303-317.

[7] Tsou, M. C., Kao, S. L., \& Su, C. M. (2010). Decision support from genetic algorithms for ship collision avoidance route planning and alerts. Journal of Navigation, 63(01), 167-182.

[8] Lazarowska, A. (2015). Ship's Trajectory Planning for Collision Avoidance at Sea Based on Ant Colony Optimisation. Journal of Navigation, 68(02), 291-307.

[9] Valero-Gomez, A., Gómez González, J. V., Garrido Bullón, L. S., \& Moreno, L. (2013). The path to efficiency: fast marching method for safer, more efficient mobile robot trajectories.

[10] Garrido S, Malfaz M, Blanco D (2013). Application of the fast marching method for outdoor motion planning in robotics. Robotics and Autonomous Systems, 61(1):106-114. 
[11] Liu, Y., \& Bucknall, R. (2015). Path planning algorithm for unmanned surface vehicle formations in a practical maritime environment. Ocean Engineering, 97, 126-144.

[12] Liu W., Motiwani A., Sharma S., Sutton R., Bucknall R. (2014), "Fault Tolerant Navigation of USV using Fuzzy Multi-sensor Fusion". Technical Report, MIDAS SMSE.2014.TR.010.

[13] Faragher R. (2012), "Understanding the Basis of the Kalman Filter Via a Simple and Intuitive Derivation". IEEE Signal Processing Magazine. Pp 128-132.
[14] Peyré, G., \& Cohen, L. (2005). Heuristically driven front propagation for geodesic paths extraction. In Variational, Geometric, and Level Set Methods in Computer Vision (pp. 173-185). Springer Berlin Heidelberg.

[15] Hart, P. E., Nilsson, N. J., \& Raphael, B. (1968). A formal basis for the heuristic determination of minimum cost paths. IEEE transactions on Systems Science and Cybernetics, 4(2), 100-107.

[16] Tsitsiklis, J. N. (1995). Efficient algorithms for globally optimal trajectories. IEEE Transactions on Automatic Control, 40(9), 1528-1538.

[17] Otsu, N. (1975). A threshold selection method from gray-level histograms. Automatica, 11(285-296), 23-27. 